\title{
Etat actuel de l'utilisation des lasers à excimères en médecine
}

\author{
S. Avrillier, E. Tinet et D. Ettori
}

\section{Laboratoire de Physique des Lasers, Universite PARIS XIII, 93430 Villetaneuse, France RESUME}

Excimer lasers are particulary relevant for microsurgery since they can produce precise cuts with very little residual thermal effect. The $308 \mathrm{~nm} \mathrm{XeCl} \mathrm{laser}$ coupled to fibers is used in cardiology and neurosurgery. The $248 \mathrm{~nm} \mathrm{KrF}$ laser can be used for the ablation of enamel in dentistry and the $193 \mathrm{~nm}$ laser is already clinically used for cornea surgery. Excimer laser-induced fluorescence is probably a good method for a medical diagnostic associated to these applications.

\section{INTRODUCTION}

Pratiquement dès que certains lasers sont devenus opérationnels et faciles à utiliser par des non spécialistes (lasers à argon, lasers à $\mathrm{CO}_{2}$, lasers YAG, lasers $\mathrm{He}-\mathrm{Ne}$ ), c'est à dire dès les années 70 , leur propriété de cohérence spatiale qui permet de concentrer leur énergie lumineuse sur de toutes petites surfaces a trouvé de nombreuses applications en médecine et en biologie. Ainsi, le laser à argon est devenu un outil presque courant pour les ophtalmologues (traitement des décollements de rétine), le laser à $\mathrm{CO}_{2}$ est utilisé comme bistouri ou pour détruire des lésions précancéreuses du col de l'utérus, le laser $\mathrm{He}-\mathrm{Ne}$ permet le positionnement spatial d'appareils de radiographie.

Au delà de ces applications immédiates, de multiples possibilités thérapeutiques et diagnostiques plus sophistiquées sont apparues au fil des années grâce à la mise en oeuvre de nouveaux lasers et grâce aux performances sans cesse accrues des fibres optiques qui permettent de véhiculer le rayonnement à l'intérieur du corps humain.

Des résultats cliniques très prometteurs ont déjà étés obtenus dans certains domaines ( réduction des plaques d'athérome en cardiologie, destruction de calcul rénaux en urologie, raccordement de petites artères en microchirurgie etc.).

L'utilisation des lasers en médecine et en biologie est donc un domaine qui devrait connaître une forte extension dans les années à venir.

De manière générale, quels que soient la longueur d'onde et le mode d'émission du laser utilisé pour une action directe sur un tissu vivant, il est intéressant de noter que l'énergie spécifique totale requise pour obtenir un résultat se situe entre 10 et $10^{3} \mathrm{~J} / \mathrm{cm}^{2}$ [1]. Les divers processus d'interaction tissu-rayonnement étant en fait déterminés par la façon dont cette énergie est délivrée. Il est d'usage de classer ces divers processus en quatre groupes par ordre de durée d'exposition qui peut, elle, varier de 12 ordres de grandeurs

-L'interaction électromagnétique obtenue avec des impulsions de 10 ps à $10 \mathrm{~ns}$.

-La photoablation U.V. (impulsions de 10 à $300 \mathrm{~ns}$ ).

-Le mode thermique ( 1 à $10 \mathrm{~s}$ d'exposition)

-L'interaction photochimique douce (quelques secondes en mode continu)

L'étendue de la zone touchée par l'irradiation dépend de la longueur d'onde d'émission du laser. Il existe pour les tissus biologiques une "fenêtre thérapeutique" incluant le visible et l'infrarouge proche ( de $400 \mathrm{~nm}$ à $1,2 \mu \mathrm{m}$ ) pour laquelle le rayonnement peut pénétrer sur plusieurs centimètres. Par contre dans l'ultraviolet (fortement absorbé par les protéines) et l'infrarouge lointain (fortement absorbé par l'eau ), la pénétration ne se fait que sur quelques dizaines de microns.

D'autres facteurs sont également importants pour déterminer l'étendue du volume affecté par l'irradiation. Dans une utilisation en mode thermique, les dimensions de ce volume dépendent des valeurs relatives du temps d'émission et du temps de diffusion thermique du tissu irradie [2]. Dans une utilisation en impulsions courtes, où cette diffusion est réduite, le volume concerné ne dépendra plus que des caractéristiques optiques du tissu (absorption, diffusion, réflexions). 
Enfin l'effet obtenu, lié à la longueur d'onde utilisée et à l'énergie accumulée à un instant donné dans un volume donné, peut aller de la simple excitation spécifique de chromophores endogènes ou exogènes contenus dans le tissu, à la destruction macroscopique d'une partie de ce volume (vaporisation, photoablation U.V. ou photodisruption). Dans la cinétique de ces processus les durées de vie des niveaux excités (électroniques, vibrationnels ou rotationnels) jouent un rôle primordial ainsi que les différentes voies de désexcitation ou les voies réactionnelles.

Parmi tous les processus utiles en médecine, le phénomène de photoablation U.V., provoqué par des impulsions de quelques dizaines de nanosecondes de lumière ultraviolette est caractérisé par l'excellente qualité et l'extrême précision des incisions obtenues.

De nombreuses études expérimentales $[3,4,5]$ ont montré qu'au dessus d'un certain seuil les lasers à excimères pouvaient être utilisés pour découper, dans pratiquement tous les matériaux biologiques, des volumes cylindriques de dimensions parfaitement contrôlées et dont les parois, exemptes de toute brûlure, sont nettes et précises: la largeur de l'incision est celle du faisceau et sa profondeur est de l'ordre de quelques dizaines de microns par impulsion laser, les tissus environnants restants intacts (figures 1 et 2)

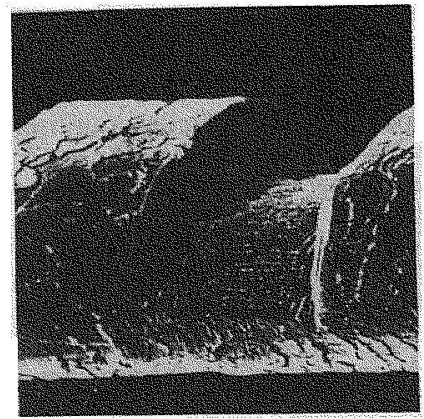

Figure 1: Cheveu humain gravé à $193 \mathrm{~nm}$

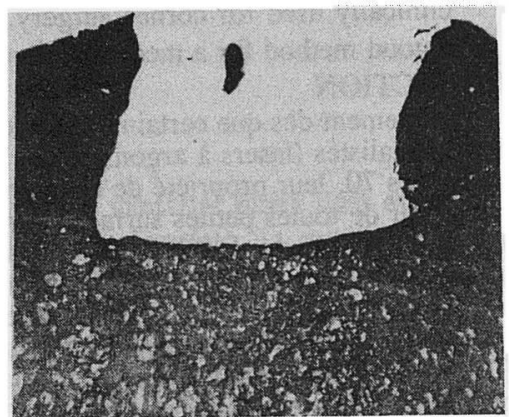

Figure 2:Impact laser obtenu en bout de fibre à $308 \mathrm{~nm}$ dans une paroi artérielle. Largeur $600 \mu \mathrm{m}$, profondeur $500 \mu \mathrm{m}$.

La "photoablation U.V." des tissus biologiques ne peut en aucun cas être comparée à une pure vaporisation thermique puisque l'énergie requise pour l'ablation d'un certain volume est typiquement 3 à 4 fois inférieure à celle que nécessiterait sa vaporisation. Comme pour les autres matériaux, ce phénomène est encore assez mal connu bien qu'il fasse l'objet de nombreuses recherches fondamentales [6]. Son étude est un domaine extrêmement riche qui demande une connaissance plus approfondie de la cinétique des transitions d'énergie des biomolécules et des effets collectifs induits dans le volume irradié.

Les applications potentielles de la photoablation U.V. sont très diverses et concernent tous les domaines où une ablation précise d'un petit volume de tissu est nécessaire. Elle est déjà utilisée à titre expérimental en ophtalmologie pour la gravure de la cornée, en cardiologie [7] pour l'ablation des plaques athéromateuses dans les artères coronaires (diamètre de l'ordre du $\mathrm{mm}$ ), en dentisterie, en neurochirurgie et de façon générale en microchirurgie (destruction de microstructures en embryologie, opérations orthopédiques fines comme celle du ménisque...).

Par ailleurs, nous verrons également que les lasers à excimères peuvent être utilisés pour établir des diagnostics par spectroscopie de fluorescence induite.

Dans l'exposé qui suit, nous évoquerons successivement les applications médicales en cours d'expérimentation ou d'évaluation dans l'ordre décroissant de leur proximité à l'utilisation clinique de routine. 


\section{OPHTALMOLOGIE}

La photoablation U.V. par laser à excimères $A r F$ à $193 \mathrm{~nm}$ est utilisée ici pour modifier la courbure de la surface de la cornée de façon à corriger des défauts de vision de l'oeil (myopie, hypermétropie, astigmatisme). Plusieurs appareils médicaux sont déjà commercialisés dans le monde entier et certains d'entre eux ont étés homologués officiellement aux Etats-Unis. Des résultats immmédiats remarquables ont étés obtenus sur plusieurs centaines de patients mais l'évolution à moyen et long terme reste encore un sujet de polémique.

Le lecteur trouvera plus de détails dans l'article de M.GROSS consacré à ce sujet dans ce même volume.

\section{CARDIOLOGIE}

Les maladies cardio-vasculaires sont une des causes de mortalité les plus fréquentes dans les pays développés. Elles sont le plus souvent provoquées par la formation de plaques athéromateuses conduisant à un rétrécissement (sténose) des artères coronaires qui irriguent le muscle cardiaque (diamètre $<3 \mathrm{~mm}$ ). Cette maladie peut également se développer dans les autres artères du corps ou artères périphériques.

L'introduction par voie percutanée fémorale d'un cathéter vecteur d'un produit radioopaque, permet de diagnostiquer de façon courante ce type d'atteintes. Lorsqu'une intervention s'impose, le chirurgien procède soit à un pontage, dans une opération à thorax ouvert (circulation extra-corporelle), soit, si cela est possible (plaque localisée et non calcifiée) à une angioplastie mécanique par un ballonnet gonflable introduit au niveau de la sténose par un cathéter du même type que celui qui est utilisé pour le diagnostic, le malade étant alors conscient. Cette technique est moins traumatique et moins chère que le pontage mais ses résultats sont souvent compromis par une resténose à court ou moyen terme. Par ailleurs ces deux méthodes ne sont pas applicables à tous les malades et en particulier dans les cas de sténoses diffuses ou/et calcifiées.

L'attrait principal de l'angioplastie par laser est qu'elle pourrait in fine être utilisée pour traiter des sténoses multiples, étendues et calcifiées des coronaires par voie percutanée. L'utilisation de lasers classiques continus (argon, YAG) fonctionnant dans le visible ou dans l'infrarouge s'est révélée jusqu'à présent assez désastreuse à cause des effets thermiques qu'il engendrent (perforations, brûlures profondes des tissus environnants, réoclusion précoce), $[8,9,10]$ Seuls les lasers pulsés restent envisageables [11] et parmi eux le laser à excimères $\mathrm{XeCl}(308 \mathrm{~nm})$ semble être le mieux placé actuellement pour diverses raisons:

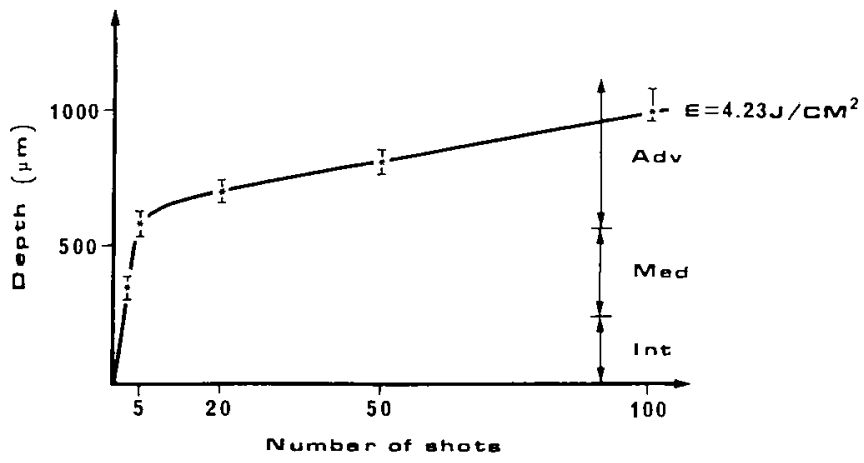

Fig. 3 - Profondeur d'ablation en fonction du nombre de tirs laser à $4.23 \mathrm{~J} / \mathrm{Cm}^{2}$ sur un fragment d'aorte humaine fortement fibrosé. L'épaisseur de chaque couche de la paroi artérielle est indiquée sur la figure ( intima (côté lumière de l'artère), média, adventice). L'efficacité de la photoablation est beaucoup plus faible dans l'adventice que dans les autres couches qui sont le siège de la maladie athéromateuse. L'adventice est atteinte en 5 tirs et la perforation n'est obtenue qu'après 100 tirs. 
a) - Les études in vitro de la photoablation à $308 \mathrm{~nm}$ de parois artérielles humaines athéromateuses dans l'air, le sérum ou le sang ont clairement démontré son aptitude à produire des incisions fines et contrôlées sans dommages thermiques sous-jacents $[3,4]$ et cela même dans des plaques fortement calcifiées. Le seuil de photoablation est voisin de 1,4 $\mathrm{J} / \mathrm{cm}^{2}$. L'épaisseur de la couche nécrosée sur les bords de l'incision est presque toujours inférieure à $50 \mu \mathrm{m}$. De plus dans tous les cas nous avons pu constater une remarquable résistance de la couche extérieure de la paroi artérielle (adventice) à la photoablation à 308 $\mathrm{nm}$ (Figure 3), ce qui représente un facteur de sécurité important pour l'angioplastie in vivo puisque la perforation de l'artère est rendue ainsi plus difficile.

b) - Il est possible, moyennant quelques précautions de transporter de fortes puissances à $308 \mathrm{~nm}$ dans des fibres optiques.

Dans ce domaine, la difficulté provient de la mauvaise qualité optique des faisceaux lasers à excimères (section rectangulaire ou carrée de 1 à $4 \mathrm{~cm} 2$ avec des inhomogénéités et des divergences horizontale et verticale différentes) et des densités surfaciques de puissance extrêmement élevées mises en jeu (de l'ordre du GigaWatt par $\mathrm{Cm} 2$ )

Les résultats que nous obtenons actuellement sont indiqués dans le tableau suivant.

Table 1 - Energies et intensités obtenues à la sortie de diverses fibres de $1 \mathrm{~m}$ de long. Durée de l'impulsion $100 \mathrm{~ns}$

\begin{tabular}{|c|c|c|}
\hline Diamètre du coeur $(\mu \mathrm{m})$ & $\begin{array}{l}\text { Energie de sortie par } \\
\text { impulsion }(\mathrm{mJ})\end{array}$ & $\begin{array}{l}\text { Int. de sortie par impulsion } \\
\left(\mathrm{J} / \mathrm{Cm}^{2}\right)\end{array}$ \\
\hline 1000 & 100 & 13 \\
600 & 48 & 17 \\
400 & 19 & 15,1 \\
200 & 4 & 12,7 \\
\hline
\end{tabular}

L'accès des artères coronaires a été récemment ouvert à l'angioplastie percutanée par l'amélioration de cathéters contenant de nombreuses fibres très fines ( $100 \mu \mathrm{m}$ de coeur) disposées concentriquement autour d'une lumière centrale. Cette lumière permet d'utiliser les mêmes guides métalliques que pour l'angioplastie par ballonnet.

Des études multicentriques visant à l'homologation de plusieurs appareils commercialisables sont en cours [12-15]. Les publications correspondantes, relatant plus de 1000 cas de par le monde, font état d'une technique sûre, grâce à laquelle des résultats immédiats satisfaisants ont étés obtenus notamment pour les lésions calcifiées. Le taux des resténoses reste voisin de celui des traitements par ballonnet ( 40 à $50 \%$ à 6 mois) mais sera très certainement réduit si la proportion de surface optiquement active est accrue à l'extrémité des cathéters.

\section{ORTHOPEDIE}

Le laser $\mathrm{XeCl}$ couplé à une fibre également été utilisé [16] pour remodeler le cartilage articulaire ou lisser le ménisque dans des opérations du genou. L'observation endoscopique permettant de travailler encore une fois à partir d'une ouverture très réduite. Plusieurs dizaines de patients ont ainsi étés traités. Bien que les résultats aient étés décrits comme satisfaisants, cette application ne semble pas, à notre connaissance, être poursuivie actuellement. 


\section{NEUROCHIRURGIE}

Des essais préliminaires, in vitro, de photoablation UV à $308 \mathrm{~nm}$ en bout de fibre de tissus cérébraux frais ont démontré qu'il était possible d'obtenir une destruction propre, sans effet thermique ni fumée, d'une région petite et bien délimitée.

Par ailleurs, les neurochirurgiens disposent actuellement d'une méthode de reconnaissance et de repérage à trois dimensions des structures cérébrales, fondée sur le traitement informatique des scans RMN. Cette méthode leur permet, grâce à la technique dite de "stéréotaxie", d'atteindre, avec une sonde très fine, n'importe quelle partie du cerveau à partir d'un orifice de dimensions réduites pratiqué dans le crâne. Le trajet en ligne droite est calculé de façon à éviter les vaisseaux sanguins. Cette opération, complètement indolore pour le patient qui n'est pas endormi, n'est utilisée actuellement que pour faire des biopsies de régions suspectes.

Une collaboration à été mise en place entre notre équipe et plusieurs équipes de neurochirurgiens, pour mettre au point une méthode thérapeutique qui, associant le laser à excimères et sa fibre UV à la technique de stéréotaxie, permettrait de détruire de petites tumeurs cérébrales sans ouvrir le crâne du malade.

Une expérimentation animale in vivosur le rat a été entamée en mai 1991 pour déterminer les paramètres optimaux de cette méthode (énergie par impulsion, cadence de tir, surface active et géométrie de la sonde). L'examen histologique des résultats à 1 jour, 10 jours, 1 mois, 6 mois montre une destruction localisée des tissus visés avec peu de lésions dans les tissus environants. La poursuite de cette étude devrait aboutir dans un proche avenir à la création d'un nouvel outil de chirurgie à "crâne fermé".

\section{DENTISTERIE}

La prévention de la carie dentaire par utilisation de fluor a permis, depuis 1965 , une récession d'environ $50 \%$ du nombre des caries sur les surfaces lisses des dents. Par contre, le nombre de caries sur les sillons des molaires reste important notamment chez les enfants. Ces sillons, de largeur très faible, peuvent atteindre la jonction entre l'émail et la dentine et les poils des brosses à dents sont trop gros pour pouvoir nettoyer correctement ces fissures. Il est donc nécessaire de trouver d'autres méthodes de prévention.

Nous avons donc décidé [17], avec le Pr. MELCER, de faire une étude in vitro de l'action, sur ces sillons, du rayonnement laser $\mathrm{KrF}$ à $248 \mathrm{~nm}$. Le but étant d'une part de réaliser un sillon plus large ( environ $1 \mathrm{~mm}$ ) et d'autre part de modifier et de stériliser l'émail au fond du sillon.

25 molaires humaines, saines, ont été irradiées avec des intensités allant de 0,5 à 13 $\mathrm{J} / \mathrm{cm}^{2}$ pendant des séquences de 200 à 500 tirs laser. La forme du faisceau a été travaillée optiquement pour obtenir des traits de 50 microns à $2 \mathrm{~mm}$ de large sur $1 \mathrm{~cm}$ de long. Chaque échantillon est ensuite observé à la loupe binoculaire puis au microscope électronique. La dent est ensuite sectionnée le long de l'impact laser pour étudier les effets en profondeur.

La photoablation réalise une gravure parfaitement linéaire avec des parois lisses et régulières. La profondeur et la largeur de l'impact laser dépend de la densité surfacique d'énergie par impulsion. Le seuil se situe à $(0,7 \pm 0,2) \mathrm{J} / \mathrm{cm}^{2}$. La profondeur d'ablation est de 0,4 micron par impulsion à $5 \mathrm{~J} / \mathrm{cm}^{2}$.

Le fond du sillon est lisse et restructuré sur environ 1 micron de profondeur, ce qui laisse penser qu'il existe alors une bonne protection contre la carie: le fond du sillon devient accessible et $\mathrm{s}^{\prime}$ est montré résistant aux solutions acides.

Des expériences sont actuellement en cours pour affiner les paramètres de l'irradiation. Cette technique pourrait dans l'avenir devenir une méthode de prévention efficace de la carie dentaire.

Par ailleurs, la photoablation UV des céramiques étant un domaine très peu exploré, nous avons entamé une étude sur des céramiques proches de l'émail. 


\section{DIAGNOSTIC}

La fluorescence induite par laser sur les molécules endogènes ou sur des colorants exogènes spécifiques est un très bon moyen de diagnostic en temps réel de l'état des cellules. Citons parmi d'autres la possibilité de mesurer ainsi le rapport NAD/NADH, révélateur de l'état d'anoxie des cellules [18], ou le diagnostic très précoce de cancer par détection de la fluorescence de l'hématoporphyrine fixé sélectivement sur des cellules malignes [19,20]. Encore une fois les fibres optiques permettent de travailler in situ par une technique non invasive. Ce domaine est encore peu exploré et la meilleure connaissance des spectres de fluorescence des divers tissus, in vivo, devrait apporter dans l'avenir beaucoup de moyens diagnostiques supplémentaires.

Il serait très intéressant, en particulier, de pouvoir utiliser la fluorescence induite par une impulsion à très faible énergie du laser à excimères thérapeutique pour savoir avant de déclencher la photoablation U.V. si le tissu situé à l'extrémité de la fibre est effectivement un tissu à détruire.

Un appareil de spectroscopie par fluorescence induite en bout de fibre, utilisant un détecteur optique multicanaux a été mis au point dans notre laboratoire. Il permet d'enregistrer un spectre de fluorescence avec un seul tir de quelques $\mu \mathrm{J}$ au bout d'une fibre de $600 \mu \mathrm{m}$ de diamêtre de coeur. Il est utilisé in vitropour la reconnaissance de l'athérome dans les coronaires, mais aussi pour tenter de différentier les tissus cérébraux traversés au cours d'une procédure de stéréotaxie; on espère ainsi disposer d'une méthode diagnostique plus rapide et mieux adaptée au traitement ablatif par laser que la méthode biopsie + analyse histologique.
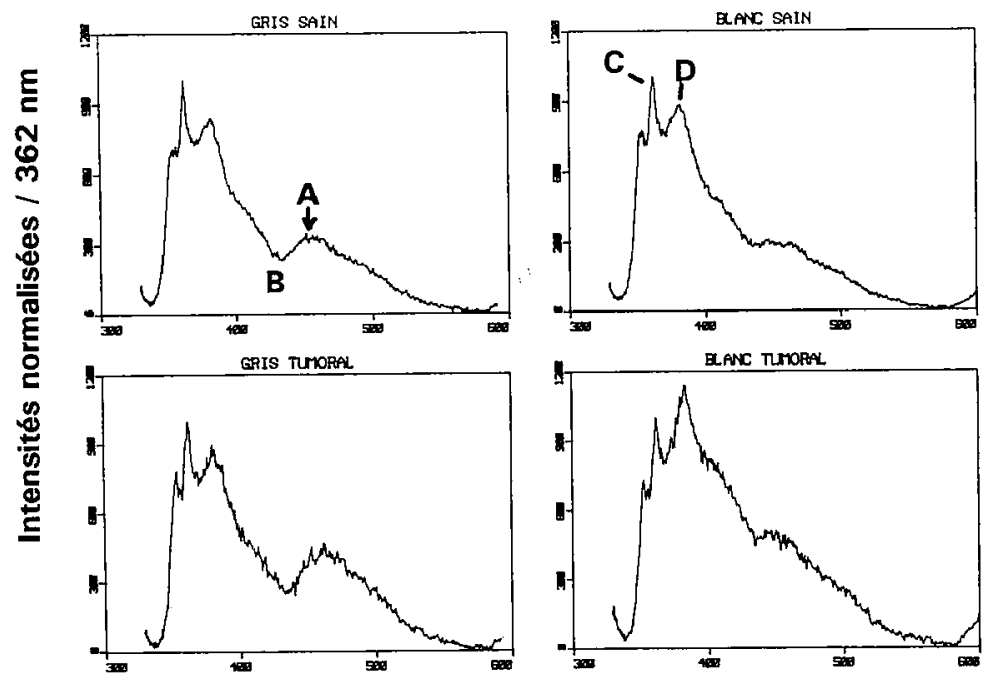

\section{Longueurs d'onde en $\mathrm{nm}$}

Figure 4: Spectres de LIF (excitation à $308 \mathrm{~nm}$ ) obtenus in vitroen bout de fibre après un seul tir de $4 \mu \mathrm{J}$ dans de la matière cérébrale humaine. La matière blanche se distingue de la matière grise grâce au rapport des intensités à 451,9 (A) et 433,9 nm (B). L'état cancéreux se traduit dans la matière blanche par l'intensité relative des pics à 362 (C) et $383,6 \mathrm{~nm}$ (D) Des tests plus fins sont à trouver en ce qui concerne l'état cancéreux de la matière grise. 
Les premiers spectres réalisés montrent une différence sensible entre les différents types de tissus. On commence ainsi à reconnaitre la matière cérébrale blanche ou grise, saine ou cancéreuse ( voir Figure 4 ).

\section{CONCLUSION}

Les méthodes de chirurgie par lasers à excimères sont encore à leur début. Associées, pour certaines d'entre elles, à un diagnostic par fluorescence induite, elles trouveront très certainement, dans un proche avenir, leur place spécifique parmi les outils thérapeutiques.

\section{BIBLIOGRAPHIE}

[1] BOULNOIS J.L.Lasers in Medical Science vol 1 (1986)

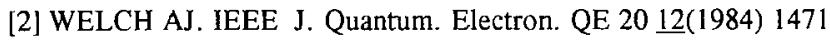

[3] LINSKER R., SRINIVASAN R., WYNNE J.J. AND ALONSO D.R., Lasers Surg. Med. 4 (1984) 201.

[4] SINGLETON D.L..PARASKEVOPOULOS G, TAYLOR R.S. HIGGISON L.A.J IEEE J. Quantum Electron. QE23 (1987) 1772.

[5] DELETTRE E., AVRILLIER S., OLLIVIER J.P., BERTHIER J.P., RAYNAL E. AND ROUGIER Y. accepted Lasers in Med. Science.

[6] LAZARE S. AND GRANIER V., Laser Chem. 10 (1989) 25.

[7] OLLIVIER J.P, AVRILLIER S, GANDJBAKHCK I, RAYNAL E, DEBOURAYNE J, DRONIOU J,Eur. Heart. J. $\underline{9}$ (1988)331

[8] CHOY D.S.J., STERTZER S.H., ROTTERDAM H.Z. AND BRUNO M.S., Am. J. Cardiol. $\underline{50}$ (1982) 1209.

[9] ABELA G.S., NORMANN S., COHEN D., FELDMAN R.L., GEISER E.A. AND CONTI R., Am. J. Cardiol. 50 (1982) 1199.

[10] OLLIVIER J.P., ROSSANT P., GANDJBAKHCH I., WARME-JANVILLE B., QUENZER A., BRION R. ET DRONIOU J., Arch. Mal. Coeur 4 (1985) 554.

[11]For an extensive bibliography, see for example SANBORN, T.A.Circulation 78 (1988) 769.

[12] LITVACK F, EIGLER N.L., MARGOLIS J.R., et al Am.J.Cardiol.66(1990)1027

[13] SANBORN T.A., BITTL J.A., HERSHMAN R.A., SIEGEL R.M.

J.Am.Coll.Cardiol. 17(1991)169B-73B

[14] EUROPEAN STUDY GROUP ON CORONARY EXCIMER LASER ANGIOPLASTY Circulation 84Supl Il(1991)362 
[15]COOK S.L., EIGLER N.L., SHEFER A., GOLDENBERG T., FORRESTER J.S,, LITVACK F.,

Circulation $\underline{84}(1991) 632$

[16| KOORT H.J., SPIE 1424(1991)53

|17| MELCER J., VITALE M., AVRILLIER S., LABORDE P., GUILLAUMIN D., Innov. Tech.Biol.Med. $11(1990) 68$

| I8| RENAULT G, RAYNAL E, SINET M, MUFFAT-JOLY M, VALLOIS J.M, BERTHIER J.P, CORNILLAULT J, GODARD B. Am. Heart. J.108(1984)428

|19| DOUGHERTY T.J, Photochem. Photobiol. 45(1987)879

|20| For an extensive hibliography se ANDERSSON-ENGELS S., Lund Reports on Atomic Physics LRAP108(1989) 